\title{
V445 PUPPIS, A FIRST IDENTIFIED HELIUM NOVA
}

\author{
Mariko Kato ${ }^{1}$ and Izumi Hachisu ${ }^{2}$
}

\section{RESUMEN}

La explosión de 2001 de V445 Puppis ha mostrado que se trata de un objeto unico que presenta ausencia de hidrógeno, enriquecimiento de helio y carbono y una lenta caída de la curva de luz con una amplitud pequeña. Hemos calculado curvas de luz teóricas de novas de helio para confirmar que V445 Pup es el primer ejemplo de una nova de helio. Del ajuste de la curva de luz podemos concluir que la enana blanca es muy masiva $\geq 1.33 M_{\odot}$.

\section{ABSTRACT}

The 2001 outburst of V445 Puppis is an unique object, that shows absence of hydrogen, enrichment of helium and carbon, slow decay of the light curve with a small amplitude. We have calculated theoretical light curves of helium novae to confirm that V445 Pup is the first example of helium nova. From the light curve fitting we conclude that the white dwarf is very massive $\geq 1.33 M_{\odot}$.

\section{Key Words: NOVAE, CATACLISMIC VARIABLES - STARS: INDIVIDUAL (V445 PUPPIS) - STARS: MASS LOSS - WHITE DWARF}

\section{INTRODUCTION}

The unique object V445 Puppis was discovered in the outburst phase on 30 December 2000 by Kanatsu (Kato \& Kanatsu 2000). Its spectrum shows absence of hydrogen, enrichments of helium and carbon. Near infrared spectra confirm the absence of Paschen and Brackett hydrogen lines (Ashok \& Banerjee 2003, and references therein). From its small amplitude of the outburst $\left(\Delta m_{\mathrm{v}} \sim 6\right)$, the shape of light curve, which does not resemble those of novae, and the spectrum feature, Ashok \& Banerjee (2003) suggested this object to be a helium nova.

Helium novae were theoretically predicted by Kato, Saio, \& Hachisu (1989). It is a nova outburst phenomenon caused by a helium shell flash on a white dwarf (WD). They considered two possible cases of helium accretion; The first case is a helium accretor from the companion helium star, and another is a hydrogen-rich matter accretor from a normal companion with a high accretion rate. In the latter case, hydrogen-rich matter accreted is processed into helium and then accumulates on the WD. When the mass of the helium layer reaches a critical mass, an unstable weak helium shell flash occurs to trigger a helium nova outburst. In such helium novae, mass loss owing to optically thick winds is relatively weak, and most of the helium envelope burns into carbon and oxygen and accumulates on the WD (Kato \& Hachisu 1999). After many periodic helium shell

\footnotetext{
${ }^{1}$ Keio University, Yokohama, Japan.

${ }^{2}$ University of Tokyo, Tokyo, Japan.
}

flashes, the WD gradually grows in mass and ends up an accretion induced collapse to a neutron star or explodes as a Type Ia supernova (Nomoto, Thielemann \& Yokoi 1984).

In this paper, we present light curve modeling of V445 Pup and show that this object is indeed a helium nova on a massive WD. A full version of this work has been found in Kato \& Hachisu (2003).

\section{THE MODEL}

The decay phase of helium nova outbursts can be followed by the optically thick wind theory (Kato \& Hachisu 1994). The structure of the WD envelope is calculated by solving the equations of motion, continuity, energy transport, and energy conservation. The details of computation have been already published in Kato \& Hachisu (2003). The WD masses are assumed to be 1.1, 1.2, 1.3, 1.33, 1.35 , and $1.377 M_{\odot}$. For the 1.1 and $1.2 M_{\odot}$ WDs, we assume the Chandrasekhar radius, and for the $1.3 M_{\odot} \mathrm{WD}$, we adopt the radius of helium burning zone, $\log R_{\mathrm{WD}}(\mathrm{cm})=8.513$ (Kato et al. 1989). For more massive WDs, we adopt the radius of accreting WDs (Nomoto, Thielemann \& Yokoi 1984). The $1.377 M_{\odot}$ WD is a limiting case of massaccreting WDs just before a central carbon ignition (Nomoto, Thielemann \& Yokoi 1984). The composition of the envelope is assumed to be uniform with $(X, Y, C+O, Z)=(0.0,0.48,0.5,0.02)$ for $\leq 1.3 M_{\odot}$, which is taken from Kato \& Hachisu (1999), and $(0.0,0.38,0.6,0.02)$ for $\geq 1.33 M_{\odot}$. Changing the ratio of carbon to oxygen with the total mass ratio 


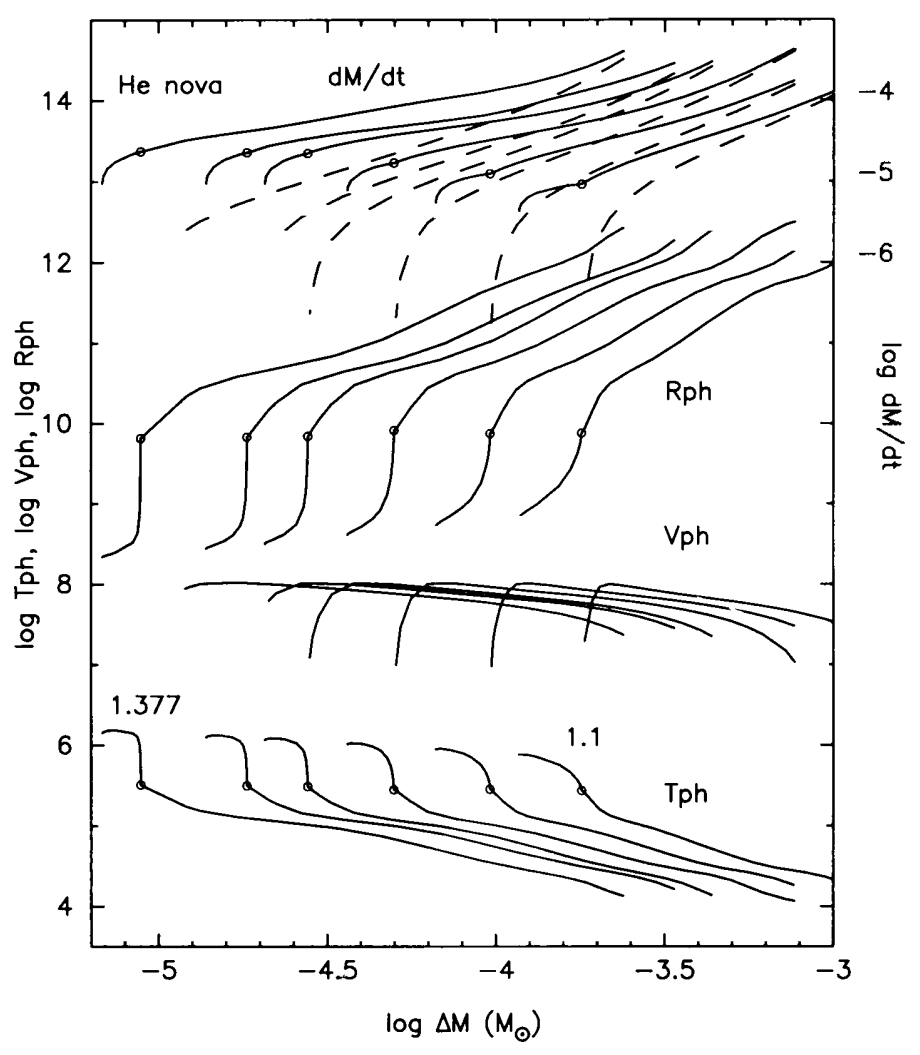

Fig. 1. Top:Total envelope mass decreasing rate (nuclear burning + wind mass loss) (solid curve), and wind mass loss rate (dashed curve) in units of $M_{\odot} \mathrm{yr}^{-1}, \mathrm{Sec}$ ond: Photospheric radius $R_{\mathrm{ph}}(\mathrm{cm})$, Third: wind velocity $V_{\mathrm{ph}}\left(\mathrm{cm} \mathrm{s}^{-1}\right)$, Bottom: temperature $T_{\mathrm{ph}}(\mathrm{K})$, against the envelope mass $(\Delta M)$ in units of $M_{\odot}$. Time runs from right to left because the envelope mass is decreasing in time. The wind mass loss stops at the point marked by a small open circle. The WD mass is $1.1,1.2,1.3,1.33$, 1.35 , and $1.377 M_{\odot}$, from right to left.

constant $(C+O=$ const. $)$ does hardly change the result. OPAL opacity is used.

\section{LIGHT CURVES}

Figure 1 shows the photospheric temperature $T_{\mathrm{ph}}$, the photospheric wind velocity $V_{\mathrm{ph}}$, the photospheric radius $R_{\mathrm{ph}}$, the wind mass loss rate and the total mass decreasing rate of the envelope (the wind mass loss rate plus the nuclear burning rate). At the peak of the optical light curve, the star reaches somewhere on the curve depending on the envelope mass, and moves leftward in time. The wind mass loss stops at the point denoted by the small open circles. After that the star further moves leftward as the envelope mass decreases owing to nuclear burning.

Theoretical light curves corresponding to the models in Figure 1 are plotted in Figure 2. A more massive WD shows a more rapid decline because of a smaller envelope mass as shown in Figure 1. Difference in the chemical composition hardly

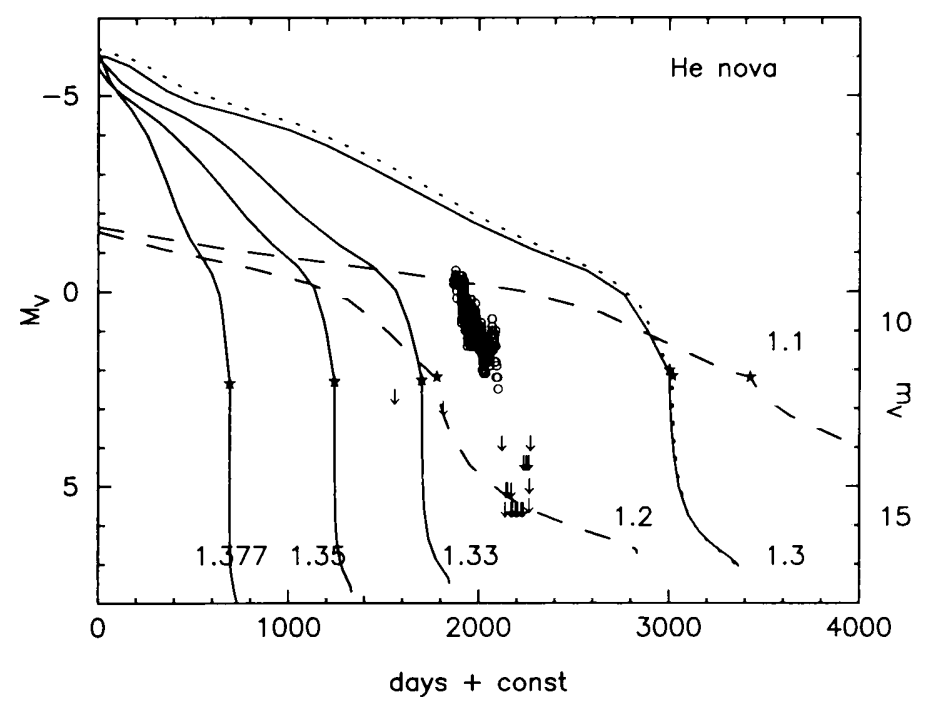

Fig. 2. Theoretical light curves for helium novae. WD masses are attached to each light curve in units of $M_{\odot}$. The dotted curve denotes a $1.3 M_{\odot}$ model with different composition (for detail, see text). The wind mass loss stops at asterisk on each curve. Dots and arrows represent observational data taken from VSNET (magnitude scale is in the right-hand side).

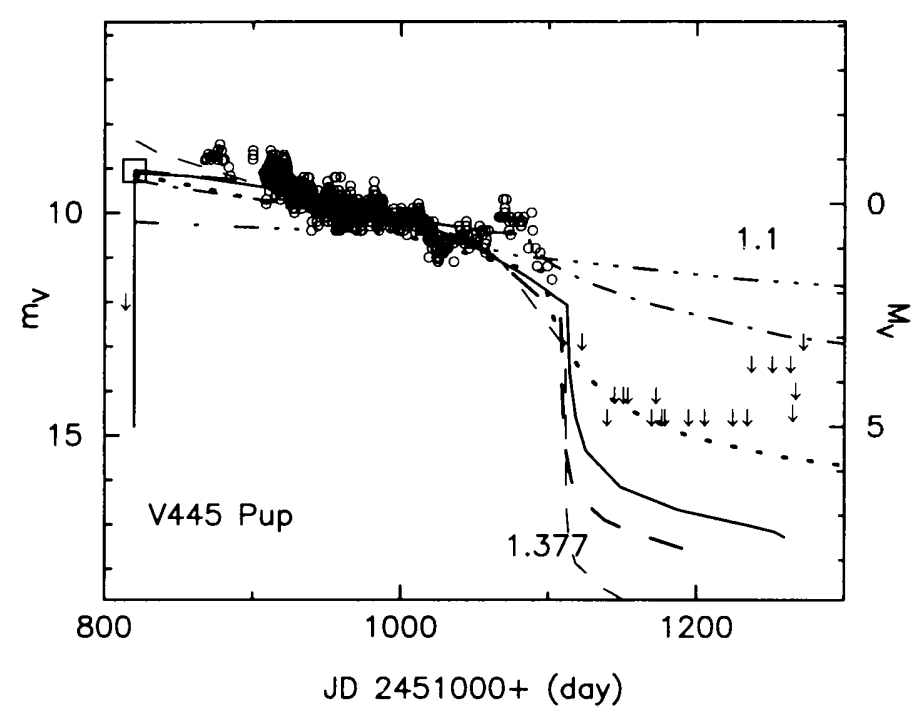

Fig. 3. Same as figure 2 but its enlargement. 1.1 (dashthree-dotted), 1.2 (dash-dotted) , 1.3 (dotted), 1.33 (solid), 1.35 (dashed), and $1.377 M_{\odot}$ (thin dashed). The theoretical curves except $1.33 M_{i}$, are shifted in the vertical direction: $1.5,1.5$ and $0.5 \mathrm{mag}$ upward for $1.1,1.2$ and $1.3 M_{\odot}$ and 0.2 and $0.9 \mathrm{mag}$ downward for 1.35 and $1.377 M_{\odot}$, respectively.

changes the theoretical light curves as shown in Figure 2 (see the difference between the dotted curve for $(Y, C+O)=(0.38,0.6)$ and the solid curve for $(0.48,0.5)$ on the $1.3 M_{\odot}$ WD).

After the onset of a helium shell flash, the star brightens up and reaches somewhere on the theoretical curve in Figure 2. Note that the time $t=0$ in these template light curves does not mean the on- 
set of ignition of a particular object. The first point is determined by the helium ignition mass, i.e., the envelope mass at helium ignition for an individual object. If the ignition mass is very massive, it appears much left-side on these light curves, and the outburst lasts for a long time. The $V$-magnitude decreases slowly in the early phase but quickly drops in the later stage. After the wind stops at the point denoted by asterisk, the star fades away very quickly. The helium burning stops at the last point of each curve.

Observational data of V445 Pup (taken from VSNET ${ }^{3}$ ) is shown in Figures 2 and 3 . The mean decreasing rate of $\mathrm{V} 445 \mathrm{P} u p$ is about $1.8 / 200$ (mag day ${ }^{-1}$ ). The light curves of the 1.1 and $1.2 \mathrm{M} \odot$ WD decline too slow to be compatible with the observation even if we choose any parts of their light curves. On the other hand, we are able to fit a part of our theoretical light curves for more massive than $1.3 N I_{\odot}$ WD to the observational data. Thus, we may conclude that V445 Pup has a WD more massive than $1.3 \mathrm{M}_{(0)}$.

To fit the vertical axis of the light curves, we obtain an apparent distance modulus of $(m-M)_{V}=$ 9.8 as shown in Figure 3. The horizontal axis (time) is also shifted to fit the data. Here we adopt a middle part of our theoretical light curves around $M_{V} \sim 1$ that has a decline rate of $\sim 1.8 / 200\left(\mathrm{mag}_{\mathrm{day}}{ }^{-1}\right)$.
Figure 3 shows an enlargement of the light curve. The theoretical curves of $1.2-1.377 M$ are almost similar until JD 2,452,100, and depart from each other after that day. The light curve of 1.1 $M$., is too slow to fit the data and the curves of 1.2 and $1.3 \mathrm{M}$. WDs do not meet the upper limit observational data after JD 2,452,100. Therefore, we conclude that V445 Pup has a massive $\left(\geq 1.33 M_{\odot}\right)$ WD.

If we assume the WD mass to be $1.33 M_{\odot}$, the ignition mass is $5 \times 10^{-5} M_{\odot}$, corresponding helium accretion rate $7 \times 10^{-7} M_{\odot} \mathrm{yr}^{-1}$ and the recurrence period is 70 years. These values suggest that the WD is growing in mass and ends up either a Type Ia supernova or an accretion induced collapse to a neutron star.

\section{REFERENCES}

Ashok, N. M., \& Banerjee, D. P. K. 2003 AAp, 409, 1007 Henden, A. A., Wagner, R. M., \& Starrfield, S. G., 2001 IAU Circ. 7729

Kato, M., Saio, H., \& Hachisu, I. 1989 ApJ, 340, 509

Kato, M., \& Hachisu, I. 1994 ApJ, 437, 802

Kato, M., \& Hachisu, I. 1999 ApJ, 513, L41

Kato, M., \& Hachisu, I. 2003, ApJ, 598, L107

Kato, T., \& Kanatsu, K. 2000, IAU Circ. 7552

Nomoto, K., Thielemann, F., \&, Yokoi, K. 1984, ApJ, 286,644

Mariko Kato: Department of Astronomy, Keio University. 4-1-1, Hiyoshi, Kouhoku-ku, Yokohama, 223-8521, Japan (mariko@educ.cc.keio.ac.jp).

Izumi Hachisu: Department of Earth Science and Astronomy, College of Arts and Sciences, University of Tokyo, Komaba, Meguro-ku. Tokyo 153-8902, Japan (hachisu@chianti.c.u-tokyo.ac.jp).

\footnotetext{
${ }^{3}$ VSNET:http://vsnet.kusastro.kyoto-

u.ac.jp/vsnet/index-j.html
} 